\title{
BMJ Open Randomised trial estimating length of endotracheal tube insertion using gestational age or nasal-tragus length in newborns: a study protocol
}

\author{
Abdul Razak (D) , ${ }^{1}$ Maheer Faden, ${ }^{1}$ Jameel Alghamdi, ${ }^{2,3}$ Abdulaziz Binmanee, ${ }^{4}$ \\ Abdullah Hawash Alonazi, ${ }^{5}$ Anas Hamdoun, ${ }^{6}$ Saud Almugaiteeb, ${ }^{1}$ \\ Waseemoddin Patel, ${ }^{1}$ Hamdi Katar, ${ }^{2}$ Fabian Lora, ${ }^{7}$ Abdullah Alismail (D) ,7 \\ Adrian Lavery, ${ }^{7}$ Ibrahim Hamama, ${ }^{1}$ Noura Alsaleem, ${ }^{1}$ Manal Alshaikh, ${ }^{4}$ \\ Lama Alrasheed, ${ }^{8}$ Omar Aldibasi ${ }^{9}$
}

To cite: Razak A, Faden M, Alghamdi J, et al. Randomised trial estimating length of endotracheal tube insertion using gestational age or nasaltragus length in newborns: a study protocol. BMJ Open 2022;12:e055628. doi:10.1136/ bmjopen-2021-055628

- Prepublication history and additional supplemental material for this paper are available online. To view these files, please visit the journal online (http://dx.doi.org/10.1136/ bmjopen-2021-055628).

Received 20 July 2021 Accepted 16 December 2021

Check for updates

(C) Author(s) (or their employer(s)) 2022. Re-use permitted under CC BY-NC. No commercial re-use. See rights and permissions. Published by BMJ.

For numbered affiliations see end of article.

Correspondence to

Dr Abdul Razak;

razakmdpaed@gmail.com

\section{ABSTRACT}

Introduction Endotracheal tube (ETT) insertion depth estimation is important for optimal placement of ETT tip and balanced ventilation of the lungs. Various methods are available to determine the ETT insertion depth. The Neonatal Resuscitation Programme recommends the gestational age and nasal-tragus length (NTL) methods for estimating ETT insertion depth during cardiopulmonary resuscitation. However, the prospective data comparing these two methods is lacking.

Methods and analysis This is an open-label multi-centre randomised controlled trial, where gestational age and NTL methods will be used to determine the initial ETT insertion depth in term and preterm infants that are less than 28 days old, requiring oral intubation in the delivery room or neonatal intensive care unit (NICU).

Sites and sample size The trial is aimed to recruit 454 infants over 3 years across tertiary level NICUs.

Outcomes The primary outcome includes an optimally positioned ETT, defined as an ETT tip between the upper border of the first thoracic vertebra and the lower border of the second thoracic vertebra. The outcome is assessed by a paediatric radiologist, who will be masked to the group assignment. Secondary outcomes are malpositioned ETT tips, pneumothorax, ETT repositioning, chronic lung disease, invasive ventilation days, and death.

Analysis Data will be analysed using the intention-totreat principle. The primary and categorical secondary outcomes will be compared using the $\chi^{2}$ test. Adjusted risk ratios of outcomes will be calculated along with $95 \%$ Cls through multivariable logistic regression analysis, including covariates deemed biologically to influence the outcomes.

Ethics and dissemination The study has been approved by the PNU Research Ethics Board (20-0148) and the respective ethical review boards of the participating centres. The results will be disseminated through conference meetings, social media platforms, and publications in scientific journals.

Trial registration number NCT04393337.
Strengths and limitations of this study

- ELEGANT trial is an open-label, multicentre trial investigating the accuracy of two Neonatal Resuscitation Programme recommended endotracheal tube (ETT) insertion depth methods.

- Term and preterm infants requiring oral intubation in NICU or delivery room will be enrolled.

- Optimally positioned ETT is the primary outcome assessed by the blinded paediatric radiologist.

- Impractical to blind interventions and investigating only oral intubation are trial limitations.

\section{INTRODUCTION}

Most infants are vigorous at birth, but some infants require assisted respiratory support, including positive pressure ventilation and endotracheal intubation. ${ }^{1}$ Endotracheal intubation is a potentially life-saving measure in infants with severe hypoxia, cardiopulmonary arrest and extremely premature lungs. Endotracheal intubation is recommended when positive pressure ventilation with a face mask does not result in clinical improvement of an infant. ${ }^{2}$ Although an endotracheal tube (ETT) is the most reliable way of providing positive-pressure breath, the critical factor determining the maximal efficacy of positive-pressure ventilation is the optimal placement of the ETT tip. ETT tip should be placed in a position that allows proportional ventilation of the lungs. A misplaced ETT tip can result in heterogeneous lung expansion, lung collapse, pneumothorax and asymmetrical surfactant distribution, which may eventually lead to chronic lung disease. ${ }^{3}$ There are various methods available to determine the initial depth of ETT. Some of these methods are based on the infant's 
birth weight, ${ }^{45}$ gestational age, anthropometric measurements ${ }^{6}$ and others include vocal cord guide ${ }^{7}$ and digital palpation methods. ${ }^{8-10}$

The most used method is based on the infant's weight. In the weight-based method, so-called Tochen's formula, the ETT insertion depth in oral intubation is obtained by adding $6 \mathrm{~cm}$ to the infant's birth weight. ${ }^{4}$ This method often overestimates the insertion depth in extremely preterm infants less than 28 weeks of gestational age. ${ }^{11}$ Researchers attempted to improve the accuracy of ETT insertion depth by modifying Tochen's method from $6 \mathrm{~cm}$ to $5 \mathrm{~cm}$ plus weight. However, these modifications were not associated with statistically significant improvement in ETT tip positions. ${ }^{5}$ Similarly, clinical trials evaluated various other methods such as the vocal cord guide method, ${ }^{7}$ digital palpation $\operatorname{method}^{8-10}$ and foot length method, ${ }^{6}$ for estimating ETT insertion depth in newborn infants, but did not find promising results. A recent systematic review and meta-analysis showed that the commonly used estimation methods for ETT insertion depth are inaccurate and unreliable. ${ }^{12}$ It also addressed the gap that the methods recommended by the Neonatal Resuscitation Programme (NRP) are not extensively studied in clinical trials. $^{2}$

NRP recommends gestational age and nasal-tragus length (NTL) methods for estimating ETT insertion depth during cardiopulmonary resuscitation of the neonate. ${ }^{2}$ The evidence to support these two NRP recommended methods is, however, limited. Two observational studies showed that using a gestational age to estimate ETT insertion depth improved the proportion of correctly placed ETT tips in newborns. ${ }^{13} 14$ However, a randomised trial found no difference between gestational age and weight-based methods for estimating ETT insertion depth in newborns. ${ }^{15}$ Similarly, observational studies report the NTL method-the distance from nasal septum tip to ear tragus $+1 \mathrm{~cm}$-for estimating ETT insertion depth improves the proportion of optimally placed ETT tips in neonates, ${ }^{16-19}$ but no difference between the NTL method and weight-based method was found in a recent randomised trial. ${ }^{20}$

To the authors' knowledge, there is no head-to-head randomised trial that compares the two NRP recommended methods, gestational age and NTL methods, for estimating ETT insertion depth in newborns. Hence, this clinical trial is designed to determine the accuracy of two methods, gestational age and NTL methods (intervention and control), recommended by the eighth edition of NRP for estimating ETT insertion depth in oral intubation in term and preterm neonates requiring endotracheal intubation (population) in neonatal intensive care unit (NICU) or delivery room (setting).

\section{METHODS}

Trial hypothesis

To determine whether estimating ETT insertion depth using gestational age rather than NTL method results in more correctly positioned ETT tips in oral intubation.

\section{Trial design}

This is a multi-centre randomised controlled trial primarily conducted in tertiary level NICUs in Saudi Arabia and extends to appropriate international tertiary level NICUs. The trial commenced in June 2020 and is aimed to recruit 454 infants over 3 years.

\section{Inclusion criteria}

- Infants born between 23 weeks 0 days and 41 weeks 6 days gestational age.

- Infants less than 28 days of life.

- Infants requiring oral intubation in the delivery room or NICU.

\section{Exclusion criteria}

- Infants with a chromosomal abnormality.

- Infants with major anomalies, including craniofacial anomalies and facial dysmorphism that may affect the NTL.

- Infants with unknown gestational age at birth.

\section{Primary outcome}

The primary outcome is the proportion of the optimally positioned ETT on the chest X-ray. ETT is considered optimally positioned if the tip of the ETT lies between the upper border of the first thoracic vertebra (T1) and the lower border of the second thoracic vertebra (T2).

\section{Secondary outcomes}

The secondary outcomes include

- ETT tip above the upper border of T1.

- ETT tip below the lower border of T2.

- Pneumothorax.

- ETT repositioning (advance or withdrawn) following the chest X-ray.

- Oxygen therapy at 28 days.

- Oxygen therapy or positive pressure support (include nasal cannula $<2 \mathrm{~L} / \mathrm{min}$ and $>30 \%$ oxygen, nasal cannula $>2 \mathrm{~L} / \mathrm{min}$ and any oxygen, continuous positive airway pressure, and any oxygen, or invasive respiratory support and any oxygen) at 36 weeks postmenstrual age.

- Duration of invasive ventilation.

- Death before discharge.

\section{Trial procedures}

\section{Informed consent}

Written consent will be obtained after the parents have been given a full verbal explanation and written description (online supplemental information 1). An explanation of the consent will be conducted with the parents in their native language. A hospital-based adult interpreter will be used wherein required. A deferred written consent (after initial verbal assent) where prior written consent is not feasible as the study does not involve additional risk or investigations to the participants and the interventions are otherwise considered as standard practice recommendations by the NRP and hospital sites. 


\section{Trial interventions}

The studied interventions in this study will be two different estimation methods for ETT insertion depth in oral intubation in neonates. The two methods are the gestational age method and the NTL method. In the gestational age method, the ETT insertion depth is obtained from the gestational age chart adapted from Kempley et al. ${ }^{14}$ In the NTL method, the ETT insertion depth is calculated based on the formula-the distance from nasal septum tip to ear tragus $+1 \mathrm{~cm}$. The details of these two estimation methods are provided in online supplemental information 2, which also illustrates the positioning of the neonate before obtaining the chest X-ray.

\section{Randomisation}

Eligible infants will be randomly assigned using a 1:1 ratio to the 'NTL method' or 'gestational age method'. The randomisation is stratified by gestational age at birth ( $<28$ weeks and $>28$ weeks) and the participating centre. The randomisation sequence is generated by an independent researcher with a computer at the website www.sealedenvelope.com hosted by King Abdullah bin Abdulaziz University Hospital (KAAUH), Saudi Arabia. The randomised sequence is integrated into the in-built randomization module within the Research Electronic Data Capture (REDCap) system by the independent researcher. Therefore, the sequence is inaccessible to the trial investigators.

\section{Allocation concealment}

Allocation will be concealed by incorporating the random permuted blocks of size 2 and 4 sequences within the REDCap system.

\section{Blinding}

This will be an open-label trial. Blinding of the clinicians, nurses, and patient caregivers is impractical. However, to minimise the bias, the method used to estimate the ETT insertion depth will not be mentioned to the patient caregivers explicitly nor recorded in the patient charts. The primary outcome assessment will be blinded by masking the consultant paediatric radiologist to the group assignment. Similarly, the consultant paediatric radiologist will determine the following secondary outcomes-ETT tip above the upper border of T1, ETT tip below the lower border of T2 and pneumothorax. The trained research assistant will determine the other secondary outcomes (ETT repositioning after the X-ray, oxygen therapy at 28 days, oxygen therapy or positive pressure support at 36 weeks postmenstrual age, duration of invasive ventilation, and death before discharge).

\section{Structure and duration of trial}

The trial aims to recruit 454 infants (see under sample size) from multiple tertiary-level NICUs over 3 years.

\section{Data collection}

Data required for the trial will be collected from the clinical notes and radiological records using the data collection forms (online supplemental information 3). No additional laboratory or blood tests will be required.

\section{Early cessation}

The trial steering committee will receive recommendations from the data monitoring committee if the trial requires early termination following the interim data analyses and evidence from relevant studies. The following measures were agreed to consider stopping the trial, wholly or partly (subgroups), after an interim analysis that will be conducted following enrolment of 200 participants.

1. An absolute difference of greater than or equal to $25 \%$ in the primary outcome between the study groups.

2. An absolute difference of less than $5 \%$ in the primary outcome between the study groups.

3. A rate of less than $20 \%$ in the primary outcome in either of the groups.

The purpose of the interim analysis is to evaluate the safety and futility rather than the efficacy. Therefore, no $\mathrm{p}$ value adjustment is proposed.

\section{Safety reporting}

Any unexpected serious events (death, any life-threatening event, any event that will prolong the hospitalisation or any event that will result in disability) will be reported to the data safety monitoring committee. The committee will evaluate the risks versus benefits associated with the study or the study interventions. The committee may recommend early cessation depending on the interim data analyses.

\section{Statistics and analysis \\ Sample size}

Our unpublished data showed using the NTL method for estimating ETT insertion depth in term and preterm infants result in $35 \%$ of correctly positioned ETT tips. The data is similar to the randomised ${ }^{20}$ and non-randomised ${ }^{21}$ studies that showed similar accuracy $(32 \%-37 \%)$ with the NTL method for estimating ETT insertion depth. With $90 \%$ power and two-sided $5 \%$ significance, to detect an absolute increase of $15 \%$ in optimally positioned ETT tips using the gestational age method, we will require 454 participants. We calculated sample size using nQuery Advisor Sample Size Calculator V.8.3.0.0. ${ }^{22}$

\section{Statistical analysis}

Data will be analysed based on the intention-to-treat principle. Univariate analyses will be performed to compare baseline demographic factors between the two groups (online supplemental information 4). A mean with standard deviation (normal data) or median with IQR (skewed data) will be obtained for continuous variables and numbers and percentages for categorical variables. Independent t-test (normal data) or Mann-Whitney $\mathrm{U}$ test (skewed data) for continuous variables and $\chi^{2}$ or Fisher exact test for categorical variables when appropriate will be used to analyse the groups. SAS V.9.4 will be used for 
the conduct of all analyses. A detailed Statistical Analysis Plan will be developed before the interim analysis.

\section{Analysis of primary outcome}

Adjusted risk ratios of a successful outcome will be calculated along with 95\% CIs. Adjusted ratios will be determined using multivariable logistic regression analysis, including covariates deemed biologically to influence the primary outcome (online supplemental information $5)$. In addition, principles of best model practices will be followed (including assessment of collinearity among included variables) and determination of the predictive ability of the model using the area under the curve. Outcomes will be reported as shown in ghost tables in online supplemental information 6 .

\section{Analysis of secondary outcomes}

Similar analyses as above will be performed for all secondary outcomes that are categorical variables. Online supplemental information 5 lists variables included in the regression models for each secondary outcome. Outcomes will be reported as shown in ghost tables in online supplemental information 6 .

\section{Handling missing data}

Missing data will be evaluated in terms of their pattern (eg, missing completely at random, missing at random, etc). All analyses will be based on a listwise deletion approach where observation with complete values will be only considered for analysis for missing completely at random. Multiple imputation approaches will be applied for variables with missing values at random to impute the missing values using the recommended method. ${ }^{23}$

\section{Quality control and assurance}

Site initiation and training

The local research team will be trained in the protocol and the trial procedures in conjunction with the local principal investigator. They will deliver the training to the site physicians, respiratory therapists and nurses. In addition, the local research team will act as a point of contact for the primary coordinating centre (KAAUH) and troubleshoot as the need arises.

\section{Data collection, confidentiality and monitoring}

We will use the data collection form (online supplemental information 3) to abstract data from patient medical records (either on paper or converted to a passwordprotected excel sheet) and will store it in locked office cabinets at participating sites or on a password-protected, encrypted USB drive. The primary study site, KAAUH, will initiate the data-sharing agreement (DSA). All the data will be entered from all participating centres into a single REDCap database, which will be managed from KAAUH once the DSA has been finalised among all centres.

Patient and public involvement

No patient involved.

\section{DISCUSSION}

This clinical trial comparing the gestational age and NTL methods will provide valuable data for clinicians determining the ETT insertion depth during oral endotracheal intubation. The findings from the clinical trial will also help address the knowledge gap in this research area and help update the NRP guidelines and recommendations. The study will also investigate the effect of gestational age on either of the methods. Though the trial is not powered for important respiratory outcomes, such as air leak, ventilation days, chronic lung disease, it will also provide comparative data assessing the impact of the estimation methods on these outcomes.

This trial is well powered for detecting the important differences between the gestational age and NTL methods for estimating ETT insertion depth. Given the large number of participants to study, we anticipate few challenges enrolling the study population. The two most significant challenges include the COVID-19 pandemic and less-invasive surfactant administration (LISA). COVID-19 pandemic-related restrictions have resulted in limited accessibility for physicians to discuss essential elements of the consent process and delayed ethical approval of additional centres. Nonetheless, we aim to keep up the enrolment with verbal assent and telephonic conversations with the parents. In addition, following LISA initiation, the rate of endotracheal intubation has come down in few study centres. Hence, additional centres from the USA are currently pending to be added as study centres for this study. In addition, these site recruitment efforts will focus on centres where LISA is not yet established.

\section{ETHICS AND DISSEMINATION}

We will conduct the trial according to The Declaration of Helsinki (amended 2008) and The International Conference of Harmonization guidelines for Good Clinical Practice (E6). Our study has been approved by the PNU Research Ethics Board (20-0148) and the respective ethical review boards of the participating centres in Saudi Arabia. Additional centres will be included following the approval by the respective institutional review boards as they are currently pending approval in the USA. We will disseminate the results to the local and international neonatal community by presenting the trial findings at various paediatric and neonatal society meetings, publishing the findings in peer-reviewed journals and disseminating them through social media platforms.

\section{Author affiliations}

${ }^{1}$ Pediatrics, King Abdullah bin Abdulaziz University Hospital, Princess Nora bint Abdul Rahman University, Riyadh, Saudi Arabia

${ }^{2}$ Pediatrics, King Fahad Hospital, AlBaha, Saudi Arabia

${ }^{3}$ Pediatrics, AlBaha University, AlBaha, Saudi Arabia

${ }^{4}$ Pediatrics, King Faisal Specialist Hospital and Research Center, Riyadh, Saudi Arabia

${ }^{5}$ Pediatrics, King Fahad Medical City, Riyadh, Saudi Arabia

${ }^{6}$ Radiology, King Abdullah Bin Abdulaziz University Hospital, Riyadh, Saudi Arabia 
${ }^{7}$ Cardiopulmonary Sciences, Loma Linda University, Loma Linda, California, USA ${ }^{8}$ Epidemiology and Biostatistics, Princess Nourah Bint Abdulrahman University, Riyadh, Saudi Arabia

${ }^{9}$ Biostatistics and Bioinformatics, King Abdullah International Medical Research Center, Riyadh, Saudi Arabia

Correction notice This article has been corrected since it was published. The funding statement has been corrected.

Contributors AR, MF, JA, HK, AB, AHA, AA, AL takes responsibility for the integrity of the data and the accuracy of the data analysis. Planning, concept and design: AR, MF. Acquisition, analysis or interpretation: AR, MF, AB, SA, WP, IH, LA, OA, MA, JA, HK, AHA, AA, AL, FL, LA, OA, AH. Drafting of the manuscript: AR, MF, AB, WP, IH, JA. Critical revision of the manuscript for important intellectual context: $A R, M F, A B, N A$, JA, HK, AHA, AA, FL, AL, MA, AH, SA. Statistical analysis: LA, OA. Obtaining funding: AR, MF, NA, SA. Administrative, technical or material support: AR, SA, WP, IH, MA, FL, $L A, O A$. Conduct, reporting and supervision: $A R, M F, A B, N A, J A, H K, A H A, A A, F L, A L$, OA, AH. Trial Steering Committee members AR, MF, AB, NA, SA, WP, OA. Data Safety Monitoring Committee members: Rayan Abdulrazaq Makhdom, Sameer Awad AlMehmadi, Shorouk Issa Mohammad Alwahsh. Research Trial Coordinator: MA.

Funding Princess Nora Bint Abdulrahman University is the source of funding. Competing interests None declared.

Patient consent for publication Not applicable.

Provenance and peer review Not commissioned; externally peer reviewed.

Supplemental material This content has been supplied by the author(s). It has not been vetted by BMJ Publishing Group Limited (BMJ) and may not have been peer-reviewed. Any opinions or recommendations discussed are solely those of the author(s) and are not endorsed by BMJ. BMJ disclaims all liability and responsibility arising from any reliance placed on the content. Where the content includes any translated material, BMJ does not warrant the accuracy and reliability of the translations (including but not limited to local regulations, clinical guidelines, terminology, drug names and drug dosages), and is not responsible for any error and/or omissions arising from translation and adaptation or otherwise.

Open access This is an open access article distributed in accordance with the Creative Commons Attribution Non Commercial (CC BY-NC 4.0) license, which permits others to distribute, remix, adapt, build upon this work non-commercially, and license their derivative works on different terms, provided the original work is properly cited, appropriate credit is given, any changes made indicated, and the use is non-commercial. See: http://creativecommons.org/licenses/by-nc/4.0/.

ORCID iDs

Abdul Razak http://orcid.org/0000-0002-6185-3694

Abdullah Alismail http://orcid.org/0000-0002-7844-8943

\section{REFERENCES}

1 Stoll BJ, Hansen NI, Bell EF, et al. Neonatal outcomes of extremely preterm infants from the NICHD neonatal research network. Pediatrics 2010;126:443-56.

2 Pediatrics AAo, Association AH. Textbook of neonatal resuscitation (NRP). 8th ed. American Academy of Pediatrics, 2021.
3 Thayyil S, Nagakumar P, Gowers H, et al. Optimal endotracheal tube tip position in extremely premature infants. Am J Perinatol 2008;25:013-16.

4 Tochen ML. Orotracheal intubation in the newborn infant: a method for determining depth of tube insertion. J Pediatr 1979;95:1050-1.

5 Tatwavedi D, Nesargi SV, Shankar N, et al. Efficacy of modified Tochen's formula for optimum endotracheal tube placement in low birth weight neonates: an RCT. J Perinatol 2018;38:512-6.

6 Embleton ND, Deshpande SA, Scott D, et al. Foot length, an accurate predictor of nasotracheal tube length in neonates. Arch Dis Child Fetal Neonatal Ed 2001;85:60F-4.

7 Gill I, Stafford A, Murphy MC, et al. Randomised trial of estimating oral endotracheal tube insertion depth in newborns using weight or vocal cord guide. Arch Dis Child Fetal Neonatal Ed 2018;103:F312-6.

8 Saboo AR, Dutta S, Sodhi KS. Digital palpation of endotracheal tube tip as a method of confirming endotracheal tube position in neonates: an open-label, three-armed randomized controlled trial. Paediatr Anaesth 2013;23:934-9.

9 Murphy MC, Donoghue VB, O'Donnell CPF. Randomised trial of estimating oral endotracheal tube insertion depth in newborns using suprasternal palpation of the tip or weight. Arch Dis Child Fetal Neonatal Ed 2020;105:196-200.

10 Jain A, Finer NN, Hilton S, et al. A randomized trial of suprasternal palpation to determine endotracheal tube position in neonates. Resuscitation 2004;60:297-302.

11 Peterson J, Johnson N, Deakins K, et al. Accuracy of the 7-8-9 rule for endotracheal tube placement in the neonate. $J$ Perinatol 2006;26:333-6.

12 Razak A, Faden M. Methods for estimating endotracheal tube insertion depth in neonates: a systematic review and meta-analysis. Am J Perinatol 2021;38:901-8.

13 Mainie P, Carmichael A, McCullough S, et al. Endotracheal tube position in neonates requiring emergency interhospital transfer. Am J Perinatol 2006;23:121-4.

14 Kempley ST, Moreiras JW, Petrone FL. Endotracheal tube length for neonatal intubation. Resuscitation 2008;77:369-73.

15 Flinn AM, Travers CP, Laffan EE, et al. Estimating the endotracheal tube insertion depth in newborns using weight or gestation: a randomised trial. Neonatology 2015;107:167-72.

16 Wang T-C, Kuo L-L, Lee C-Y. Utilizing nasal-tragus length to estimate optimal endotracheal tube depth for neonates in Taiwan. Indian J Pediatr 2011;78:296-300.

17 Shukla HK, Hendricks-Munoz KD, Atakent Y, et al. Rapid estimation of insertional length of endotracheal intubation in newborn infants. $J$ Pediatr 1997;131:561-4.

18 Whyte KL, Levin R, Powls A. Clinical audit: optimal positioning of endotracheal tubes in neonates. Scott Med J 2007;52:25-7.

19 Priyadarshi M, Thukral A, Sankar MJ, et al. 'Lip-to-Tip' study: comparison of three methods to determine optimal insertion length of endotracheal tube in neonates. Eur J Pediatr 2021;180:1459-66.

20 Uygur Özgün, Öncel MY, Şimșek GK, et al. Is nasal septum-tragus length measurement appropriate for endotracheal tube intubation depth in neonates? A randomized controlled study. Am J Perinatol 2021;38:728-33.

21 Voraruth C, Tongsawang N, Ruangwattanapaisarn N, et al. Nasaltragus length for estimating optimal insertion depth of endotracheal tube in Thai neonates. J Perinatol 2020;40:595-9.

22 nQuery. Sample Size and Power Calculation [program]. 8.3.0.0 version: "Statsols". Cork, Ireland: Statistical Solutions Ltd, 2017.

23 Rubin DB. Multiple imputation for nonresponse in surveys. John Wiley \& Sons, 2004. 